\title{
Distributed Switch and Stay Combining (DSSC) with a Single Decode and Forward Relay
}

\author{
Diomidis S. Michalopoulos, Student Member, IEEE, and George K. Karagiannidis, Senior Member, IEEE
}

\begin{abstract}
A distributed version of switch and stay combining is proposed for relaying systems that utilize a single decode and forward relay. The performance analysis of such system is presented, along with that of a simplified version of the socalled incremental relaying protocol. Numerical results show that these systems achieve the beneficial effects of diversity, although they do not employ any diversity combiner at the destination terminal.
\end{abstract}

Index Terms-Distributed switched and stay combining (DSSC), relaying channel, decode and forward relaying.

\section{INTRODUCTION}

A CHIEVING spatial diversity through the use of relaying transmission is a promising, recently evolved concept that serves as a substitute of the common diversity techniques, especially when transmitting or receiving from multiple antennas is unfeasible. For the case of single relay usage, several cooperation protocols were proposed in [1]. Among those, the protocol called "incremental relaying" was shown to offer the lowest outage probability, by utilizing feedback from the destination in order to activate the relay only when the quality of the source-destination channel is not sufficiently high. According to this protocol, however, a maximal ratio combiner (MRC) needs to be employed at the destination, which renders it difficult to implement in cases where lowcomplexity hardware is required.

In this letter, we propose and analyze the performance of a distributed switch and stay combining (DSSC) scheme, which utilizes a single decode and forward (DF) relay and actually represents a virtual, distributed version of the well-known switch and stay combining (SSC) technique, used in diversity receivers [2]-[3]. Additionally, we study the performance of a similar, yet different cooperation scheme, which we call simplified incremental relaying (S-IR), closely related to the corresponding protocol proposed in [1]. The main characteristic of both systems is that they do not employ any diversity combiner at the destination terminal; they attain the beneficial effects of diversity by utilizing appropriate feedback sent by the destination.

\section{System Model And Mode OF Operation}

Consider a source node $S$ communicating with a destination one, denoted by $D$, with the aid of a single relaying node

Manuscript received January 4, 2007. The associate editor coordinating the review of this letter and approving it for publication was Prof. Alexander Haimovich. This work was partially funded by the European Union-European Social Fund, and the GSRT of the Hellenic Ministry of Development, under the project PENED 2003.

The authors are with the Electrical and Computer Engineering Department, Aristotle University of Thessaloniki, GR-54124, Greece (email: \{dmixalo, geokarag\}@auth.gr).

Digital Object Identifier 10.1109/LCOMM.2007.070018. which is represented by $R$. In particular, the destination can receive either directly from the source or indirectly, through the virtual $S-R-D$ link. In the sequel, these two paths through which communication is achieved are termed direct and relayed branch, are denoted by $\mathcal{D}$ and $\mathcal{R}$, and their corresponding instantaneous signal-to-noise ratios (SNRs) are denoted by $\gamma_{\mathcal{D}}$ and $\gamma_{\mathcal{R}}$, respectively. Further, the instantaneous SNRs of the $S-R$ and the $R-D$ channels are represented by $\gamma_{S R}$ and $\gamma_{R D}$, respectively. In case of receiving through $\mathcal{R}$, the typical time-divisioned uncoded DF protocol is employed [1]; that is, each transmission slot is divided into two subslots: In the first subslot the source transmits its data to the relay, which demodulates, remodulates and then retransmits the resultant data to the destination during the second subslot.

The point where the DSSC system differs from the typical cooperative ones that utilize a single relay, is the fashion of determining the active branch. Specifically, this system can be thought of as a virtual SSC scheme, where the two input branches are the direct and the relayed branch, respectively. To be more precise, in each transmission slot the destination compares the received SNR (which equals to either $\gamma_{\mathcal{D}}$ or $\gamma_{\mathcal{R}}$, depending on which was the active branch in the previous slot) with a preselected fixed switching threshold, denoted by $T$. If the received SNR is lower than $T$, then a branch-switching occurs. This is implemented by appropriate feedback sent to both the source and the relay, indicating a switching on the transmission path (from the direct to the relayed one and vice versa) during the next slot. Needless to say, the transmission slots are considered small enough so that constant fading conditions during two consecutive slots are assumed. Moreover, we emphasize that no diversity combiner is utilized at the destination, i.e., only one of the two possible input branches is active; this active branch is denoted by $\mathcal{B}$.

The S-IR scheme operates similarly to the incremental relaying protocol described in [1]; the destination sends request-to-forward to the relay only if the $S$ - $D$ channel is not sufficiently high, i.e., if $\gamma_{\mathcal{D}}<T$. However, terminal $D$ does not utilize a MRC in this model, hence in cases where the relayed branch is active $D$ receives only via the relayed path, and not directly from $S$. Finally, we assume that all channels (i.e., $S-D, S-R, R-D$ ) experience independent, flat and slow Rayleigh fading, with average SNRs denoted by $\bar{\gamma}_{S D}, \bar{\gamma}_{S R}$ and $\bar{\gamma}_{R D}$, respectively.

\section{Performance Analysis}

In DF relaying, the signal that reaches the destination through the relayed branch undergoes two demodulations in cascade. Thus, the equivalent SNR of the relayed branch is not trivially derived. One option is to define $\gamma_{\mathcal{R}}$ according 
to the outage performance of the relayed branch, so that the cumulative density function $(\mathrm{CDF})$ of $\gamma_{\mathcal{R}}$ evaluated at the outage threshold SNR $\gamma_{t h}^{\mathcal{R}}=2^{2 r}-1$ (where $r$ denotes the target spectral efficiency) coincides with the outage probability of the relayed branch, i.e., $\operatorname{Pr}\{\mathcal{O} \mid \mathcal{B}=\mathcal{R}\}=F_{\gamma_{\mathcal{R}}}\left(\gamma_{t h}^{\mathcal{R}}\right)$, where $\mathcal{O}$ denotes the outage event and $F_{Z}(\cdot)$ stands for the $\mathrm{CDF}$ of the random variable (RV) $Z$. Therefore, considering the fact that $\operatorname{Pr}\{\mathcal{O} \mid \mathcal{B}=\mathcal{R}\}$ is equal to the probability of the union of the outage events associated with the $S-R$ and the $R-D$ links, respectively, $\gamma_{\mathcal{R}}$ is defined as

$$
\gamma_{\mathcal{R}}:=\min \left(\gamma_{S R}, \gamma_{R D}\right)
$$

and this is the choice of the equivalent SNR that we adopt in the sequel. We emphasize here, however, that $\gamma_{\mathcal{R}}$ is the quantity associated with the relayed branch that is compared with $T$, and does not generally represent the equivalent SNR of that branch with the strict sense (i.e., if BPSK modulation is used, $\operatorname{Pr}\left\{\mathcal{E} \mid \mathcal{B}=\mathcal{R}, \gamma_{\mathcal{R}}\right\} \neq \frac{1}{2} \operatorname{erfc}\left(\sqrt{\gamma_{\mathcal{R}}}\right)$, where $\mathcal{E}$ represents the bit-error event) ${ }^{1}$.

1) Outage Probability: Let us denote with $p_{\mathcal{D}}, p_{\mathcal{R}}$ the steady-state selection probabilities of activating $\mathcal{D}$ and $\mathcal{R}$, respectively, (i.e., $p_{\mathcal{D}}=\operatorname{Pr}\{\mathcal{B}=\mathcal{D}\}, p_{\mathcal{R}}=\operatorname{Pr}\{\mathcal{B}=\mathcal{R}\}$ ), which have been evaluated in [3, eqs. (6) and (7)] as

$$
\begin{aligned}
& p_{\mathcal{D}}=\frac{F_{\gamma_{\mathcal{R}}}(T)}{F_{\gamma_{\mathcal{D}}}(T)+F_{\gamma_{\mathcal{R}}}(T)}=\frac{1-e^{-T\left[\frac{1}{\bar{\gamma}_{S R}}+\frac{1}{\bar{\gamma}_{R D}}\right]}}{2-e^{-\frac{T}{\bar{\gamma}_{S D}}}-e^{-T\left[\frac{1}{\bar{\gamma}_{S R}}+\frac{1}{\bar{\gamma}_{R D}}\right]}} \\
& p_{\mathcal{R}}=\frac{F_{\gamma_{\mathcal{D}}}(T)}{F_{\gamma_{\mathcal{D}}}(T)+F_{\gamma_{\mathcal{R}}}(T)}=\frac{1-e^{-\frac{T}{\bar{\gamma}_{S D}}}}{2-e^{-\frac{T}{\bar{\gamma}_{S D}}}-e^{-T\left[\frac{1}{\bar{\gamma}_{S R}}+\frac{1}{\bar{\gamma}_{R D}}\right]}} .
\end{aligned}
$$

Moreover, let $\gamma_{t h}^{\mathcal{D}}$ represent the outage threshold SNR of the direct branch, i.e., $\gamma_{t h}^{\mathcal{D}}=2^{r}-1$, which can be expressed in terms of $\gamma_{t h}^{\mathcal{R}}$ through $\gamma_{t h}^{\mathcal{R}}=\gamma_{t h}^{\mathcal{D}}\left(\gamma_{t h}^{\mathcal{D}}+2\right)$. Then, the outage probability of the DSSC system is derived as

$$
\begin{aligned}
\operatorname{Pr}\{\mathcal{O}\}= & p_{\mathcal{D}}\left[\left(1-F_{\gamma_{\mathcal{D}}}(T)\right) \operatorname{Pr}\left\{\mathcal{O} \mid\left(\mathcal{B}=\mathcal{D}, \gamma_{\mathcal{D}}>T\right)\right\}\right. \\
& \left.+F_{\gamma_{\mathcal{D}}}(T) \operatorname{Pr}\{\mathcal{O} \mid \mathcal{B}=\mathcal{R}\}\right] \\
& +p_{\mathcal{R}}\left[\left(1-F_{\gamma_{\mathcal{R}}}(T)\right) \operatorname{Pr}\left\{\mathcal{O} \mid\left(\mathcal{B}=\mathcal{R}, \gamma_{\mathcal{R}}>T\right)\right\}\right. \\
& \left.+F_{\gamma_{\mathcal{R}}}(T) \operatorname{Pr}\{\mathcal{O} \mid \mathcal{B}=\mathcal{D}\}\right]
\end{aligned}
$$

Note that the conditional outage probabilities in (4) are taken from the general form

$\operatorname{Pr}\left\{\mathcal{O} \mid\left(\mathcal{B}=\mathcal{Z}, \gamma_{\mathcal{Z}}>w\right)\right\}=\left\{\begin{array}{cl}\frac{F_{\gamma_{\mathcal{Z}}}\left(\gamma_{t h}^{\mathcal{Z}}\right)-F_{\gamma_{\mathcal{Z}}}(w)}{1-F_{\gamma_{\mathcal{Z}}}(w)}, & w \leq \gamma_{t h}^{\mathcal{Z}} \\ 0, & w>\gamma_{t h}^{\mathcal{Z}}\end{array}\right.$

by substituting $\mathcal{Z}$ and $w$ with the appropriate values, i.e., $\mathcal{Z} \in\{\mathcal{D}, \mathcal{R}\}, w \in\{T, 0\}$.

Additionally, the outage probability of the S-IR scenario will be given by

$$
\begin{aligned}
\operatorname{Pr}\{\mathcal{O}\}= & \left(1-F_{\gamma_{\mathcal{D}}}(T)\right) \operatorname{Pr}\left\{\mathcal{O} \mid\left(\mathcal{B}=\mathcal{D}, \gamma_{\mathcal{D}}>T\right)\right\} \\
& +F_{\gamma_{\mathcal{D}}}(T) \operatorname{Pr}\{\mathcal{O} \mid \mathcal{B}=\mathcal{R}\} .
\end{aligned}
$$

\footnotetext{
${ }^{1}$ The equivalent SNR, $\gamma_{\mathcal{R}}$, could be also defined as the SNR that leads to the same error performance, however this results in cumbersome mathematical analysis and thus it is not adopted here. Nonetheless, in the high SNR region this equivalent SNR is identical with $\gamma_{\mathcal{R}}$ as defined in (1) (see [4, Property 1]).
}

2) Bit Error Probability (BEP) Analysis: Working similarly, the BEP of the DSSC scheme can be expressed as

$$
\begin{aligned}
\operatorname{Pr}\{\mathcal{E}\}= & p_{\mathcal{D}}\left[\left(1-F_{\gamma_{\mathcal{D}}}(T)\right) \operatorname{Pr}\left\{\mathcal{E} \mid\left(\mathcal{B}=\mathcal{D}, \gamma_{\mathcal{D}}>T\right)\right\}\right. \\
& \left.+F_{\gamma_{\mathcal{D}}}(T) \operatorname{Pr}\{\mathcal{E} \mid \mathcal{B}=\mathcal{R}\}\right] \\
& +p_{\mathcal{R}}\left[\left(1-F_{\gamma_{\mathcal{R}}}(T)\right) \operatorname{Pr}\left\{\mathcal{E} \mid\left(\mathcal{B}=\mathcal{R}, \gamma_{\mathcal{R}}>T\right)\right\}\right. \\
& \left.+F_{\gamma_{\mathcal{R}}}(T) \operatorname{Pr}\{\mathcal{E} \mid \mathcal{B}=\mathcal{D}\}\right] .
\end{aligned}
$$

For the BPSK modulation, the conditional BEP (conditioned on the $\operatorname{SNR}, \gamma)$ is defined as $\operatorname{Pr}\{\mathcal{E} \mid \gamma\}=$ $1 / 2 \operatorname{erfc}(\sqrt{\gamma})$. Additionally, the relayed branch leads to an error if an error on either the $S-R$ or the $R-D$ link (but not on both) occurs. Therefore, the conditional BEP corresponding to the relayed branch, conditioned on the events that $\mathcal{B}=\mathcal{R}$ and $\gamma_{\mathcal{R}}>\omega$, where $\omega$ can take any non-negative value, is obtained by averaging over the RVs $\gamma_{S R}$ and $\gamma_{R D}$ as

$$
\begin{aligned}
& \operatorname{Pr}\left\{\mathcal{E} \mid\left(\mathcal{B}=\mathcal{R}, \gamma_{\mathcal{R}}>\omega\right)\right\} \\
= & \frac{1}{2 \bar{\gamma}_{S R}} I\left(\frac{1}{\bar{\gamma}_{S R}}, 1, \omega\right)+\frac{1}{2 \bar{\gamma}_{R D}} I\left(\frac{1}{\bar{\gamma}_{R D}}, 1, \omega\right) \\
& -\frac{1}{2 \bar{\gamma}_{S R} \bar{\gamma}_{R D}} I\left(\frac{1}{\bar{\gamma}_{S R}}, 1, \omega\right) I\left(\frac{1}{\bar{\gamma}_{R D}}, 1, \omega\right),
\end{aligned}
$$

where the auxiliary function $I(\cdot, \cdot, \cdot)$ is defined as

$$
\begin{aligned}
I(\alpha, \beta, \omega) & =\int_{\omega}^{\infty} \exp (-\alpha x) \operatorname{erfc}(\sqrt{\beta x}) d x \\
& =\frac{e^{-\alpha x} \operatorname{erfc}(\sqrt{\beta \omega})}{\alpha}-\frac{\sqrt{\beta} \operatorname{erfc}(\sqrt{(\alpha+\beta) \omega})}{\alpha \sqrt{\alpha+\beta}} .
\end{aligned}
$$

For the analytical derivation of (8) the reader is referred to Appendix. Likewise, the conditional error probability $\operatorname{Pr}\left\{\mathcal{E} \mid\left(\mathcal{B}=\mathcal{D}, \gamma_{\mathcal{D}}>\omega\right)\right\}$ can be expressed as

$$
\operatorname{Pr}\left\{\mathcal{E} \mid\left(\mathcal{B}=\mathcal{D}, \gamma_{\mathcal{D}}>\omega\right)\right\}=\frac{1}{2 \bar{\gamma}_{S D}} I\left(\frac{1}{\bar{\gamma}_{S D}}, 1, \omega\right) .
$$

Consequently, a closed-form expression for the BEP is derived by simply plugging (2), (3), (7) and (9) in (6). Note that the probabilities $\operatorname{Pr}\{\mathcal{E} \mid \mathcal{B}=\mathcal{R}\}$ and $\operatorname{Pr}\{\mathcal{E} \mid \mathcal{B}=\mathcal{D}\}$ can also be expressed as shown respectively in (7) and (9), by setting $\omega=$ 0.

Regarding the S-IR protocol, the BEP is given by

$$
\begin{aligned}
\operatorname{Pr}\{\mathcal{E}\}= & \left(1-F_{\gamma_{\mathcal{D}}}(T)\right) \operatorname{Pr}\left\{\mathcal{E} \mid\left(\mathcal{B}=\mathcal{D}, \gamma_{\mathcal{D}}>T\right)\right\} \\
& +F_{\gamma_{\mathcal{D}}}(T) \operatorname{Pr}\{\mathcal{E} \mid \mathcal{B}=\mathcal{R}\} .
\end{aligned}
$$

Hence, the BEP for this case is derived in closed form by plugging (7) and (9) in (10).

3) Spectral Efficiency: The expected spectral efficiency of the DSSC scheme, defined as the average spectral efficiency viewed in a long-term perspective, is expressed as

$$
\widetilde{r}=p_{\mathcal{D}} r+p_{\mathcal{R}} \frac{r}{2},
$$

where $r$ denotes the spectral efficiency of the direct transmission (i.e., where no relaying takes place). Likewise, the expected spectral efficiency of the S-IR scheme is expressed as [1]

$$
\widetilde{r}=\left(1-F_{\gamma_{\mathcal{D}}}(T)\right) r+F_{\gamma_{\mathcal{D}}}(T) \frac{r}{2} .
$$




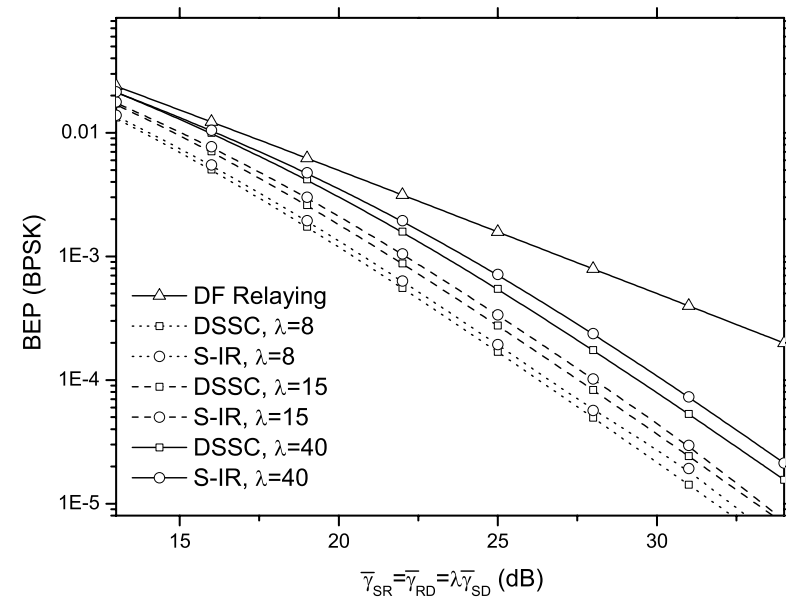

Fig. 1. Average BEP of the DSSC and the S-IR scheme for several channel realizations, assuming BPSK modulation

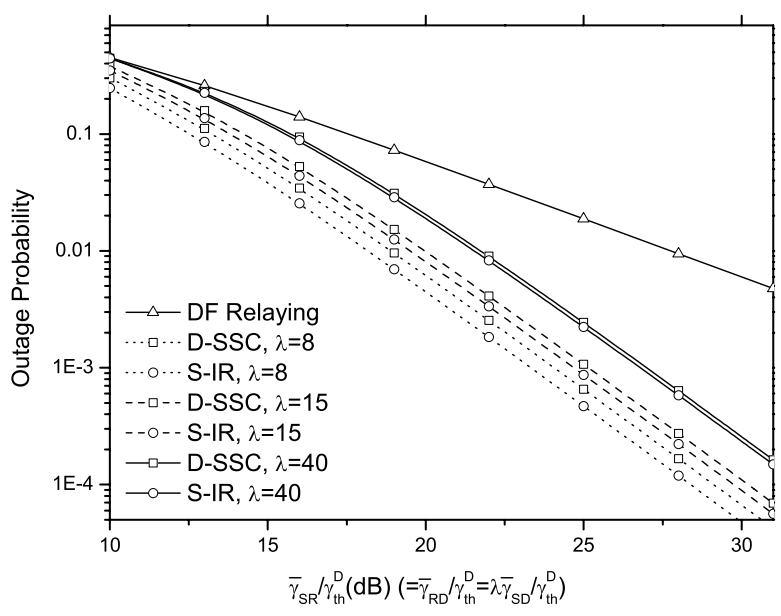

Fig. 2. Outage probability of the DSSC and the S-IR scheme for several channel realizations

\section{NUMERICAL RESULTS AND DISCUSSION}

In this section, we present some numerical examples demonstrating the BEP and outage performance of the proposed DSSC and the S-IR scheme, for several channel realizations. Specifically, the $S-R$ and $R-D$ channels are assumed to experience independent, identically distributed Rayleigh fading, with average SNR equal to $\lambda$-times the average SNR of the $S$-D link, i.e., $\bar{\gamma}_{S R}=\bar{\gamma}_{R D}=\lambda \bar{\gamma}_{S D}$, where $\lambda \in$ $\{8,15,40\}$.

Fig. 1 depicts the BEP of the proposed DSSC and the SIR scheme, versus $\bar{\gamma}_{S R}$, assuming BPSK modulation. Each curve in Fig. 1 was generated by using the optimal switching threshold $T$, which is derived numerically by minimizing (6) and (10) with respect to $T$. In general, the BEP performances of these two systems are close with one another; however, the DSSC scheme offers a lower BEP, which becomes more evident as the $S-D$ channel becomes relatively bad. Loosely speaking, this can be explained by considering the fact that in the DSSC scheme the relayed branch is used for a greater fraction of time, hence the higher the $\bar{\gamma}_{S R}$ and $\bar{\gamma}_{R D}$ (compared to $\left.\bar{\gamma}_{S D}\right)$, the greater the difference in the corresponding BEPs.

In Fig. 2, we plot the outage probability of the previous two schemes versus the normalized value of $\bar{\gamma}_{S R}$ with respect to $\gamma_{t h}^{\mathcal{D}}$, for several $\lambda$ assumptions. Similarly to the previous figure, we used the numerically-derived optimal $T$ to generate the corresponding curves. As expected, the S-IR scheme slightly outperforms the DSSC one in terms of outage probability, since the relayed branch is less frequently used and thus the expected spectral efficiency is higher (see eq. (11) and (12)).

Observing the slope of the curves in both figures, it is evident that both the DSSC and the S-IR scheme attain diversity order of two. This can be also verified by taking the limits of (6) and (10) in the asymptotically high SNR region, which is omitted here for brevity. We also note that the curves labeled as "DF relaying" in both figures correspond to the case where $D$ receives only through the $\mathcal{R}$ (and never through the $\mathcal{D}$ ) branch, where, obviously, unitary diversity order is attained. Finally, we note that the results presented here were also verified by simulations, however the simulation curves are omitted so as not to reduce the figures' clarity.

As a conclusion, we would like to point out that the DSSC scenario studied in this letter represents only a small part of the concept of virtual switched diversity systems. Extending this concept in cases where more relays of several types are used, is a challenging future-research topic.

\section{APPENDIX}

Using integration by parts, we obtain

$$
\begin{aligned}
\int e^{-\alpha x} \operatorname{erfc}(\sqrt{\beta x}) d x= & \frac{1}{\alpha}\left[-e^{-\alpha x} \operatorname{erfc}(\sqrt{\beta x})\right. \\
& \left.-\int e^{-\alpha x}\left(\frac{\beta e^{-\beta x}}{\sqrt{\pi} \sqrt{\beta x}}\right) d x\right],
\end{aligned}
$$

where we have used the integral representation of the error function, $\operatorname{erf}(\cdot)$, given in [5, eq. 8.251.1] to infer the derivative of $\operatorname{erfc}(\cdot)$. Using [5, eq. 8.251.1] again, (13) yields

$$
\begin{aligned}
& \int e^{-\alpha x} \operatorname{erfc}(\sqrt{\beta x}) d x \\
= & -\frac{e^{-\alpha x} \operatorname{erfc}(\sqrt{\beta x})}{\alpha}-\frac{\sqrt{\beta} \operatorname{erf}(\sqrt{(\alpha+\beta) x})}{\alpha \sqrt{\alpha+\beta}} .
\end{aligned}
$$

Consequently, (8) is straightforwardly derived from (14).

\section{REFERENCES}

[1] J. N. Laneman, D. N. C. Tse, and G. W. Wornell, "Cooperative diversity in wireless networks: efficient protocols and outage behavior," IEEE Trans. Inf. Theory, vol. 50, pp. 3062-3080, Dec. 2004.

[2] A. A. Abu-Dayya and N. C. Beaulieu, "Analysis of switched diversity systems on generalized-fading channels," IEEE Trans. Commun., vol. 42, pp. 2959-2966, Nov. 1994.

[3] C. Tellambura, A. Annamalai, and V. K. Bhargava, "Unified analysis of switched diversity systems in independent and correlated fading channels," IEEE Trans. Commun., vol. 49, pp. 1955-1965, Nov. 2001.

[4] T. Wang, A. Cano, G. B. Giannakis, and J. N. Laneman, "Highperformance cooperative demodulation with decode-and-forward relays," IEEE Trans. Commun., to appear.

[5] I. S. Gradshteyn and I. M. Ryzhik, Table of Integrals, Series, and Products, Sixth Ed. New York: Academic, 2000. 